\title{
Array model of shock pressure sensor for shooting point detection
}

\author{
Changlong Zhou, Yingjun $\mathrm{Li}^{*}$, Guicong Wang, and Xue Yang \\ School of Mechanical Engineering, University of Jinan, Jinan, China
}

\begin{abstract}
The array model of double- $T$ shock pressure sensor is established. Shock wave is produced by a supersonic object in the air. Pressure is produced in the process of shock wave transmission. Different shock pressure sensors have different time to receive the pressure signal. In this paper, the shooting point calculation model and the finite element model of the double T-shaped array method are established. The simulation experiment is carried out. The law of shock wave propagation is verified. The model can be used to calculate the coordinates of shooting point quickly. This method is suitable for small angle oblique fire location problem, and improves the detection accuracy of shooting point.
\end{abstract}

Keywords: Target surface, Shock pressure sensor, Acoustic positioning, Double T-type.

\section{Introduction}

In order to improve the efficiency of training, it is necessary to detect the coordinates of hitting target quickly and accurately in shooting competition. The detection methods of bullet hitting target surface position include coordinate target based on image processing, metal rod measuring target, linear CCD photoelectric target, photoelectric coordinate detection target and sound locating target [1-3]. Among them, the acoustic location detection technology has been widely used because of its strong adaptability to environment and convenient arrangement. The arrays of shock pressure sensor in sound positioning detection are N-point line array, L-type array, double triangle array and T-type array [4-6]. The method of sound positioning is characterized by convenient installation, strong environmental adaptability, reliable performance, small size, low cost and simple structure.

In this paper, the double-t type array model and mathematical model are established. The solution method of double-T model is described. The feasibility of the model is verified by simulating the shock wave produced by the bullet.

\footnotetext{
* Corresponding author: me liyj@ujn.edu.cn
} 


\section{Establish positioning model}

In this paper, the layout model of double-T shock pressure sensor is proposed. In addition, the algorithm of the model is also proposed. The double T-type array model is composed of two single T-shaped arrays. The first single T-type array consists of $Q_{4}, Q_{5}, Q_{6}$ and $Q_{7}$. The second single T-type array consists of $Q_{1}, Q_{2}, Q_{3}$ and $Q_{5}$. Two single T-types array share the same shock pressure sensor $Q_{5}$. Seven shock pressure sensors are arranged on the same level. The time difference signal detected by the shock pressure sensor of the first single Tarray and the shock pressure sensor of the second single T-array are solved jointly. In this way a more accurate bullet velocity can be measured. This method can improve the accuracy of hitting point detection. It also detects the flight angle and trajectory at the end of the bullet. It has high detection accuracy for oblique projectiles.

When an object flies in the air at supersonic speed, it will produce a strong disturbance faster than the speed of sound. The air around the projectile produces compression and expansion, which forms shock wave. The change of shock pressure curve can be divided into four processes. In the first process, the shock wave generated by the supersonic flying object increases from the air pressure $P_{0}$ to $P_{0}+P_{1}$ in a very short time $\tau_{1}$. In the second process, the pressure drops to atmospheric pressure $P_{0}$. In the third process, the projectile flying over causes the ambient air pressure to decrease from atmospheric pressure $P_{0}$ to $P_{0^{-}}$ $P_{1}$. In the third process, the projectile flying over causes the atmospheric pressure to decrease from $P_{0}$ to $P_{0}-P_{1}$. In the fourth process, the projectile flies past the sensor and the pressure returns to atmospheric pressure $P_{0}$. It is often called $\mathrm{N}$-wave because of the "N" shape of the air pressure. The pressure signal received by the sensor is shown in figure 1 , in which $T_{F}$ is the width of $\mathrm{N}$ wave. The characteristics of double $\mathrm{T}$ model include the following steps. The three-dimensional space coordinate system is established, with sensor $Q_{2}$ as the origin, the direction parallel to the target plane as the $X$ axis, the vertical direction as the $Y$ axis, and the direction perpendicular to the target plane as the $Z$ axis. Sensor position coordinates: $Q_{1}\left(-\mathrm{L}_{1}, 0,0\right), Q_{2}(0,0,0), Q_{3}\left(L_{1}, 0,0\right), Q_{4}\left(-L_{1}, 0, L_{2}\right), Q_{5}\left(0,0, L_{2}\right)$, $Q_{6}\left(L_{1}, 0, L_{2}\right), Q_{7}\left(0,0,2 L_{2}\right) . M$ is the location of the hit. The location of the sensor is shown in figure 2 .

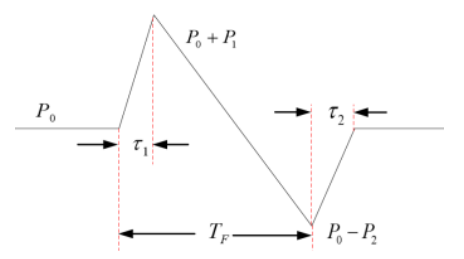

Fig. 1. $\mathrm{N}$ wave diagram.

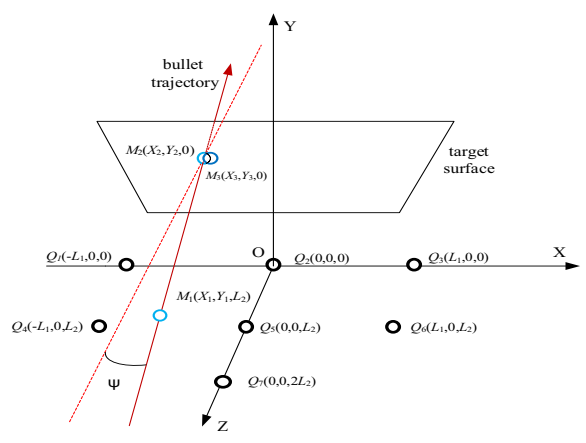

Fig. 2. Sensor position. 
In shooting competition, the shock wave first reaches the first single T-shaped array. Suppose that the first coordinate point is detected as $M_{1}$. The four shock pressure sensors $Q_{4}, Q_{5}, Q_{6}$ and $Q_{7}$ receive the time signals of bullets passing by, which are $T_{4}, T_{5}, T_{6}$ and $T_{7}$,respectively. Suppose the coordinates of $M_{1}$ is $\left(X_{1}, Y_{1}, L_{2}\right)$. It is known that the horizontal distance between $Q_{4}, Q_{5}$ and $Q_{6}$ sensors is $L_{1}$, and the distance between $Q_{7}$ and $Q_{5}$ is $L_{2}$. The solution equation is as follows.

$$
\begin{aligned}
& {\left[\left(X_{1}+L_{1}\right)^{2}+Y_{1}^{2}\right]^{1 / 2}-\left(X_{1}^{2}+Y_{1}^{2}\right)^{1 / 2}=v_{p 1}\left(t_{4}-t_{5}\right)} \\
& {\left[\left(X_{1}-L_{1}\right)^{2}+Y_{1}^{2}\right]^{1 / 2}-\left(X_{1}^{2}+Y_{1}^{2}\right)^{1 / 2}=v_{p 1}\left(t_{6}-t_{5}\right)}
\end{aligned}
$$

Bullet speed.

$$
v_{1}=\frac{L_{2}}{t_{5}-t_{7}}
$$

Sound velocity at ambient temperature.

$$
c=331.3+0.606 t
$$

Propagation velocity of shock wave.

$$
v_{p 1}=v_{1} \tan \left(\sin ^{-1} \frac{c}{v_{1}}\right)
$$

According to the equations (1), (2), (3), (4), the equations are established, and the values of $X_{1}$ and $Y_{1}$ are obtained. The coordinate point $M_{1}$ detected in the first T-shaped array is $\left(X_{1}, Y_{1}, L_{2}\right)$. Then the shock wave reaches the second single T-shaped array. Suppose that the coordinate point is detected as $M_{2}$. The time for $Q_{1}, Q_{2}, Q_{3}$ and $Q_{5}$ sensors to receive the pressure signal is $T_{1}, T_{2}, T_{3}$ and $T_{5}$, respectively. Suppose the coordinates of $M_{2}$ is $\left(X_{2}, Y_{2}, 0\right)$. It is known that the horizontal distance between $Q_{1}, Q_{2}$ and $Q_{3}$ sensors is $L_{1}$, and the distance between $Q_{2}$ and $Q_{5}$ is $L_{2}$. The solution equation is as follows.

$$
\begin{aligned}
& {\left[\left(X_{2}+L_{1}\right)^{2}+Y_{2}^{2}\right]^{1 / 2}-\left(X_{2}^{2}+Y_{2}^{2}\right)^{1 / 2}=v_{p 2}\left(t_{1}-t_{2}\right)} \\
& {\left[\left(X_{2}-L_{1}\right)^{2}+Y_{2}^{2}\right]^{1 / 2}-\left(X_{2}{ }^{2}+Y_{2}^{2}\right)^{1 / 2}=v_{p 2}\left(t_{3}-t_{2}\right)}
\end{aligned}
$$

The speed of the bullet.

$$
v_{2}=\frac{L_{2}}{t_{2}-t_{5}}
$$

The velocity of the shock wave.

$$
v_{p 2}=v_{2} \tan \left(\sin ^{-1} \frac{c}{v_{2}}\right)
$$

According to the equations (3), (5), (6), (7), the equations are established, and the values of $X_{2}$ and $Y_{2}$ are obtained. The coordinate point $M_{2}$ detected in the second T-shaped array is $\left(X_{2}, Y_{2}, 0\right)$. The distance between $M_{1}$ and $M_{2}$. 


$$
\left[\left(X_{1}-X_{2}\right)^{2}+\left(Y_{1}-Y_{2}\right)^{2}+\left(L_{2}-0\right)^{2}\right]^{1 / 2}=L_{3}
$$

Substitute $L_{3}$ into formula (10). The corrected velocity $V_{3}$ is obtained. In order to correct the coordinate position, $V_{3}$ is introduced into formula (11) to obtain the shock propagation velocity.

$$
\begin{aligned}
& {\left[\left(X_{3}+L_{1}\right)^{2}+Y_{3}^{2}\right]^{1 / 2}-\left(X_{3}{ }^{2}+Y_{3}^{2}\right)^{1 / 2}=v_{p 3}\left(t_{1}-t_{2}\right)} \\
& {\left[\left(X_{3}-L_{1}\right)^{2}+Y_{3}^{2}\right]^{1 / 2}-\left(X_{3}{ }^{2}+Y_{3}^{2}\right)^{1 / 2}=v_{p 3}\left(t_{3}-t_{2}\right)}
\end{aligned}
$$

The velocity of bullet after correction.

$$
v_{3}=\frac{L_{3}}{t_{2}-t_{5}}
$$

The velocity of shock wave after correction.

$$
v_{p 3}=v_{3} \tan \left(\sin ^{-1} \frac{c}{v_{3}}\right)
$$

where: $V_{1}, V_{2}$ are bullet velocity, $C$ is local sound velocity, $V_{P 1}, V_{P 2}$ are shock wave propagation velocity.

According to the equations (3), (9), (10), (11), the equations are established, and the values of $X_{3}$ and $Y_{3}$ are obtained. The corrected coordinate point $M_{3}$ is $\left(X_{3}, Y_{3}, 0\right)$. The detection accuracy can be improved by this method.

\section{Simulation experiment}

The shock pressure sensors $Q_{1}, Q_{2}, Q_{3}$ and $Q_{4}, Q_{5}, Q_{6}$ are equally spaced in a straight line. The distance $L_{1}$ between two adjacent sensors is $10 \mathrm{~cm}$. The shock pressure sensors $Q_{4}, Q_{5}$ and $Q_{6}$ are equally spaced in front of $Q_{1}, Q_{2}$ and $Q_{3}$, respectively. Shock pressure sensor $Q_{7}$ is located in front of $Q_{5}$. The location is shown in figure 3 .

When an object moves faster than the speed of sound. The disturbance in the air cannot reach the front end of the flying body. The gas is compressed into a concentrated stream. Compressed gases produce compression and expansion. The conical shock wave formed at this time is shock wave effect. The cone shock wave is shown in figure 4.

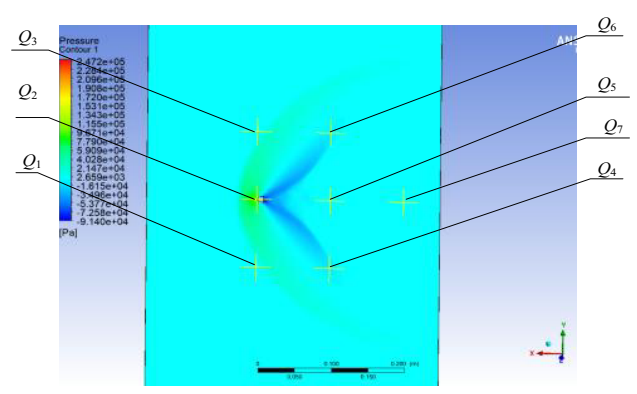

Fig. 3. sensor location diagram.

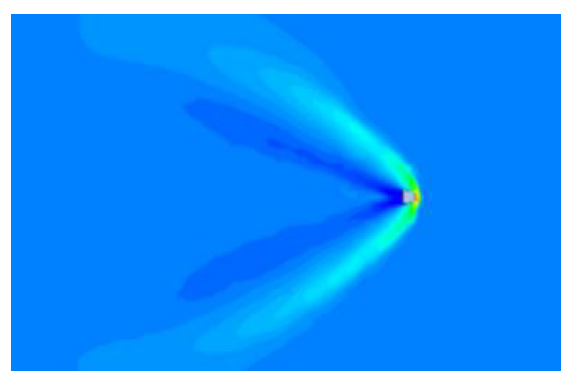

Fig. 4. conical shock.

Simulation software can be used to simulate the supersonic motion of objects in the air. The pressure signal is received by the sensor. The signals received by shock pressure sensors $Q_{1}, Q_{2}, Q_{3}, Q_{4}, Q_{5}, Q_{6}$ and $Q_{7}$ are shown in figures 5 . 


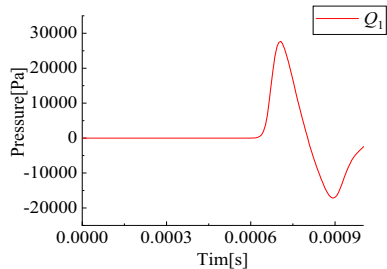

(a) $Q_{1}$

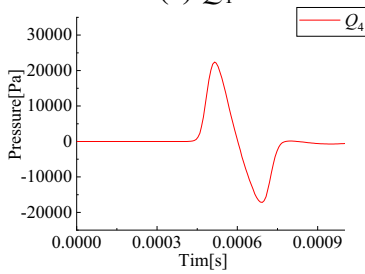

(d) $Q_{4}$

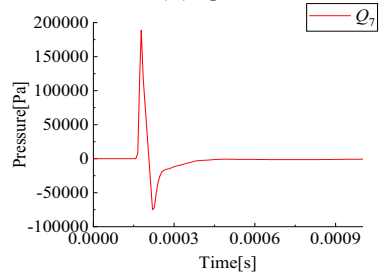

(g) $Q_{7}$

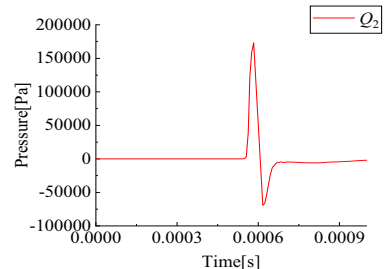

(b) $Q_{2}$

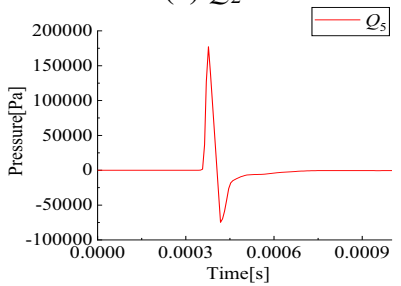

(e) $Q_{5}$

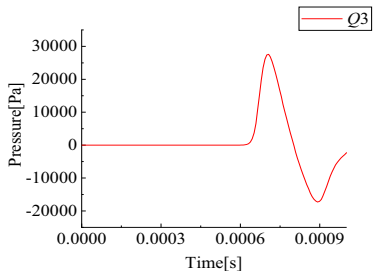

(c) $Q_{3}$

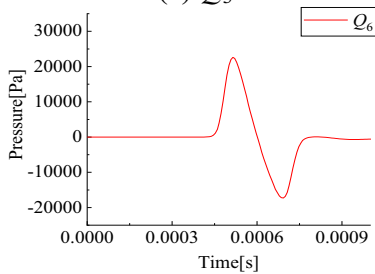

(f) $Q_{6}$

Fig. 5. Each point pressure signal.

From the above figures, it can be seen that the pressure signal received by the shock pressure sensor is in the form of "N". The time points of peaks and troughs can be obtained from the figures. The median time of $\mathrm{N}$ wave is taken as the time when the sensor receives the pressure signal. Table 1 shows the arrival time of pressure signal.

Table 1. Sensors position and time signals.

\begin{tabular}{ccc}
\hline Sensor & Coordinate(cm) & Median time(s) \\
\hline$Q_{1}$ & $(24,10,0)$ & 0.000739 \\
$Q_{2}$ & $(24,0,0)$ & 0.000593 \\
$Q_{3}$ & $(24,-10,0)$ & 0.000740 \\
$Q_{4}$ & $(14,10,0)$ & 0.000500 \\
$Q_{5}$ & $(14,0,0)$ & 0.000346 \\
$Q_{6}$ & $(14,-10,0)$ & 0.000499 \\
$Q_{7}$ & $(4,0,0)$ & 0.000093 \\
\hline
\end{tabular}

\section{Conclusions}

Based on the traditional N-line array. In this paper, the structure of double T-type array method is adopted. The mathematical model of target reporting system is obtained.

By analysing the movement of objects in the air. The $\mathrm{N}$-wave diagram of the sensor is obtained. The arrival time of $\mathrm{N}$ wave is obtained by the sensors. There will be a pitch angle when shooting at an oblique angle. The shooting angle can also be calculated on the basis 
of the double T-type mathematical model. Due to the limited space, this paper does not give the solution of oblique fire. It will be introduced in another article.

This work is supported by a grant from the National Natural Science Foundation of China (Grant No. 51875250), A Project of Shandong Province Higher Educational Youth Innovation Science and Technology Program (Grant No. 2019KJB018), National Natural Science Foundation of China (Grant No. 51705200).

\section{References}

1. Li Y J and Yang Z K 2020 Research on piezoelectric pressure sensor for shock wave load measurement ISA Transactions $\mathrm{p} 384$

2. Wang G C and Li Y J 2021 Acceleration self-compensation mechanism and experimental research on shock wave piezoelectric pressure sensor Mechanical Systems and Signal Processing p 107303

3. Nie K and Zeng K J 2018 Impact Point Quickly Calculation Method for Aircraft Based on Regular Shape Simulation Target Command Control \& Simulation p 128

4. Yang X S and Yin W J 2018 Feasibility Analysis on the Survey Scheme of Fall Point at Sea Command Control \& Simulation $\mathrm{p} 134$

5. Wang Y and Hongkai L I Research on coordinate positioning model of supersonic projectile target Journal of Applied Acoustics p 942

6. Lou J S and Wei H E 2017 Research on an improved algorithm based on TDOA for the location of cannonball impact points Electronic Design Engineering p 126 